\title{
Evaluation of Macular Microcirculation in Healthy Children Using Optical Coherence Tomography Angiography
}

\author{
(1) Yalcin Karakucuk, ${ }^{1}$ (1) Aysegul Comez,, ${ }^{2}$ () Abdullah Beyoglu, ${ }^{2}$ () Dilek Yasa ${ }^{3}$ \\ ${ }^{1}$ Department of Ophthalmology, Selcuk University Faculty of Medicine, Konya, Turkey \\ 2Department of Ophthalmology, Kahramanmaras Sutcu Imam University Faculty of Medicine, Kahramanmaras, Turkey \\ ${ }^{3}$ Department of Ophthalmology, University of Health Science, Beyoglu Eye Training and Research Hospital, Istanbul, Turkey
}

\begin{abstract}
Objectives: This study was designed to evaluate macular perfusion in a healthy pediatric population using optical coherence tomography angiography (OCTA).

Methods: Fifty-three eyes of 30 healthy children (20 boys and 10 girls) were included in this prospective, cross-sectional study. Age, gender, foveal thickness (FT), superficial foveal avascular zone area (FAZs), deep foveal avascular zone area (FAZd), superficial capillary plexus flow area (SCP), deep capillary plexus flow area (DCP), choriocapillaris flow area (CC), vascular density in the SCP (VDs), vascular density in the DCP (VDd), and subfoveal choroidal thickness (SFCT) were evaluated.

Results: The mean age was $12.0 \pm 4.0$ years (range: $6-15$ years). Gender did not have a statistically significant influence on the FAZs or FAZd. However, there was a positive correlation between the $C C$ and age $(r=0.31 ; p=0.025)$. The FAZs and FAZd were negatively correlated with the FT $(p<0.00 I)$. The VDd was positively correlated with the FT $(p=0.03)$ and was negatively correlated with the FAZd $(p=0.019)$.

Conclusion: The present study determined normative data of OCTA parameters in healthy children and provides useful insight into the relationship between age, macular microvascular networks, and the FAZ.
\end{abstract}

Keywords: Children, foveal avascular zone, optical coherence tomography angiography, vascular density

\section{Introduction}

Optical coherence tomography angiography (OCTA) is a rapid and noninvasive means to evaluate the retina and choroidal vascular structures. It is based on the principle of decorrelation, in which the red blood cell signal fluctuation can be seen on B-scan images in each specific area of the retina. OCTA demonstrates the retinal vascular structures layer by layer, thereby facilitating differentiation of the su- perficial capillary plexus (SCP), deep capillary plexus (DCP), and choriocapillaris (CC). OCTA facilitates better diagnosis and treatment as well as offering important information for greater understanding of several anatomical and physiological conditions ( 1 ).

Several studies have reported the use of this technique to evaluate the retinal vasculature and estimate the FAZ area in children (2-6). Enhanced knowledge of normal OCTA values

How to cite this article: Karakucuk Y, Comez A, Beyoglu A, Yasa D. Evaluation of Macular Microcirculation in Healthy Children Using Optical Coherence Tomography Angiography. Beyoglu Eye J 202I; 6(I): 43-48.

Address for correspondence: Yalcin Karakucuk, MD. Selcuk Universitesi Tip Fakultesi, Goz Hastaliklari Anabilim Dali, Konya, Turkey Phone: +90 5360652075 E-mail: drkarakucuk83@gmail.com

Submitted Date: July 17, 2020 Accepted Date: December 25, 2020 Available Online Date: February 16, 2021

${ }^{\circ}$ Copyright 2021 by Beyoglu Eye Training and Research Hospital - Available online at www.beyoglueye.com OPEN ACCESS This work is licensed under a Creative Commons Attribution-NonCommercial 4.0 International License. 
in children will help guide the diagnosis and treatment of many diseases, as well as understanding physiological changes. A few studies have reported the characteristics of the FAZ and vessel density in healthy eyes needed to validate OCTA findings in abnormal eyes. However, the effect of sex and age on OCTA parameters is inconsistent in these reports (6-9). Also, these studies were performed with different OCTA platforms, and racial differences in the FAZ have been reported in pediatric patients (8). Thus, there is still need for better definition of OCTA parameters in pediatric eyes.

The objective of this study was to assess the normal values of OCTA parameters in healthy children with an RTVue$X R$ Angiovue imaging system (software version: 20I5.I.0.90; Optovue, Inc., Fremont, CA, USA). Gender-specific differences in macular perfusion were also examined. Analysis of potential relationships between these vital parameters was the secondary focus of the present study.

\section{Methods}

This prospective, observational, cross-sectional study was conducted at a tertiary hospital. The research work was evaluated and authorized by the Institutional Ethics Committee (no: 235) (Protocol no: 235/31.07.2019). Written, informed consent was obtained from the parents or guardians. The Declaration of Helsinki and other international guidelines were observed throughout the research.

Healthy subjects in the age group of 6 to 15 years were recruited for the study. A detailed clinical examination was conducted for the participants as part of the eligibility and primary patient evaluation. Regular evaluation of the uncorrected and corrected distance visual acuity was performed using the Snellen chart and followed by a slit-lamp examination, cycloplegic refraction testing, and dilated fundus examination. Participants with any history of ocular disease or those who had undergone any kind of intraocular surgery or therapeutic intervention were not included. Medication use for more than a month during the eligibility assessment also disqualified a study candidate. Since refractive error can influence the outcome of the OCTA measurements, patients with a spherical or cylindrical refractive error of $>2 D$ were also excluded from participation. Similarly, those with ocular inflammation and diseases, such as uveitis, or any kind of intervention in the vitreous or retinal vascular network or any nearby region, were not considered for this study. Children with poor cooperation (unreliable examination) or less than high-quality images (signal strength index $<70$ ) were also eliminated from the research.

\section{OCTA Technique}

High-quality images of the retinal structure and assessment of the associated retinal vessels were obtained using a spec- tral-domain OCTA system. The RTVue-XR Angiovue OCTA imaging system uses a split-spectrum, amplitude-decorrelation angiography algorithm to examine blood flow and generate data.

The device performs 70.000 A-scans per second. Three-dimensional OCTA scans were obtained over $6 \times 6$ $\mathrm{mm}$ regions with 5 repeated B-scans at 216 raster positions. Every B-scan contained 216 A-scans that were arranged in the transverse direction as a $304 \times 304-p i x e l$ grid. Each scan was completed in approximately 3 seconds. The automatic segmentation feature was used for intraretinal layer segmentation, which defined the outer retinal, superficial capillary plexus (SCP), deep capillary plexus (DCP), and choriocapillaris (CC) layers. All of image scanning and estimations was performed by experienced operators.

The parameters evaluated were age, gender, foveal thickness (FT), superficial foveal avascular zone area (FAZs), deep foveal avascular zone area (FAZd), SCP flow area, DCP flow area, CC flow area, vascular density in the SCP (VDs), vascular density in the DCP (VDd), and the subfoveal choroidal thickness (SFCT). The area pertaining to the flow area of the SCP and DCP was automatically calculated using AngioVue software, as presented in Figure Ia and in Figure lb. OptoVue software was used to measure the CC flow area. The vessel density was estimated according to the percentage of the region covered by vessels and the microvessels. The mean VD of both the SCP and DCP was computed by the software, as shown in Figure $2 a$ and Figure $2 b$.

\section{Statistical Analysis}

The data were analyzed using SigmaStat 3.5 software (Systat Software Inc., San Jose, CA, USA). The Kolmogorov-Smirnov test was used to test the normality of the dataset. Continuous data were presented as mean $\pm S D$. Between-group analyses based on sex were performed using the Mann-Whitney $\mathrm{U}$ test. The Spearman correlation coefficient was used to investigate correla $\neg$ tion between OCTA parameters and age. A univariate linear regression test was conducted to study the effect of FT on VD and FAZ in the SCP and DCP. A binomial sign test was used to calculate the power of the study. A p value of $<0.05$ was considered statistically significant for all the analyses.

\section{Results}

A total of 53 eyes of 30 pediatric subjects were analyzed. Seven children with poor cooperation could not be examined in detail and the quality of the images of 7 eyes was poor. Binomial testing indicated an intermediate level power $(0.17)$ for this study of 53 eyes $(0.80=[1-\beta]$ and $\alpha=0.05)$. The results constitute a set of normative values for OCTA parameters in a healthy pediatric population. The observed 


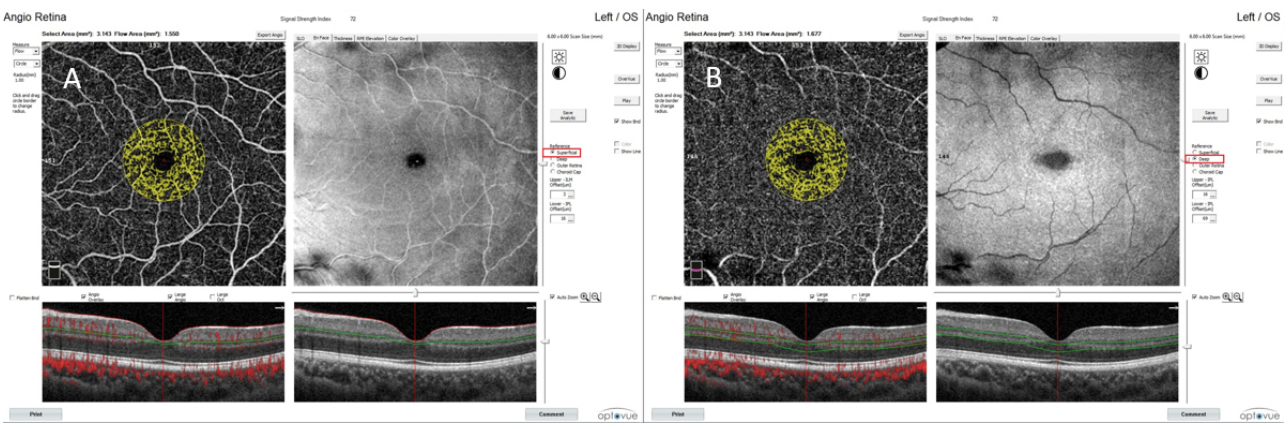

Figure I. The flow area of the (a) superficial capillary plexus and (b) the deep capillary plexus as automatically calculated using AngioVue software.

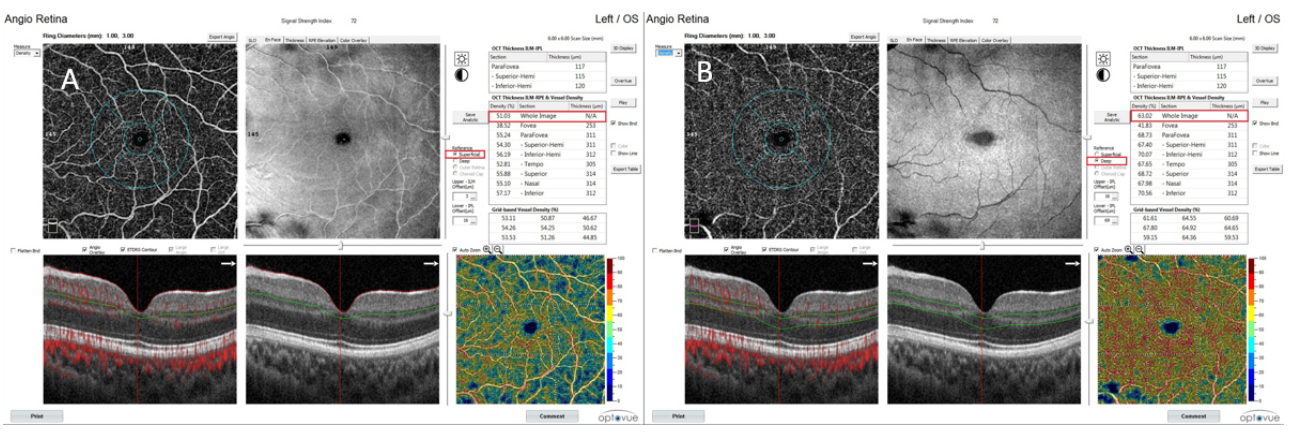

Figure 2. The mean vascular density as computed in the (a) superficial capillary plexus and (b) the deep capillary plexus by AngioVue software.

values of each parameter measured are presented in Table I. The male-to-female ratio in this study was 20:10. The mean age of the participants was $12.0 \pm 4.0$ years (range: 6 - I5 years). Statistical evaluation of the values obtained for each parameter was performed with particular attention to the sex of the subjects. The findings suggested that sex had no statistically significant effect on OCTA parameters $(p<0.05)$
The results are displayed in Table I.

The CC was positively correlated with age and the result was found to be statistically significant $(r=0.3 \mathrm{I}$; $p=0.025$ ) (Table 2). The analysis of other parameter relationships are presented in Table 3 . The results suggested that the FAZs and FAZd were negatively correlated with the FT $(p<0.00 I)$. In contrast, the VDd was positively cor-

Table I. Normative data of optical coherence tomography angiography parameters for the study group and based on gender

Characteristics

Total group n=30, 53 eyes Mean \pm SD

\section{Gender distribution}

p

\section{Male, $n=20,34$ eyes}

Mean士SD

\begin{tabular}{lcc}
\hline Age, years & $12.0 \pm 4.0$ & $12 \pm 2$ \\
SCP flow area, $\mathrm{mm}^{2}$ & $14.5 \pm 0.90$ & $14.5 \pm 0.94$ \\
DCP flow area, $\mathrm{mm}^{2}$ & $15.8 \pm 1.13$ & $15.7 \pm 1.05$ \\
CC flow area, $\mathrm{mm}^{2}$ & $19.5 \pm 0.58$ & $19.4 \pm 0.5$ \\
FAZs, $\mathrm{mm}^{2}$ & $0.24 \pm 0.13$ & $0.26 \pm 0.1$ \\
FAZd, $\mathrm{mm}^{2}$ & $0.31 \pm 0.10$ & $0.29 \pm 0.1$ \\
VDs, \% & $53.4 \pm 2.17$ & $53.4 \pm 1.5$ \\
VDd, $\%$ & $60.3 \pm 2.80$ & $60.2 \pm 2.73$ \\
CMT, $\mu \mathrm{m}$ & $222.0 \pm 26.3$ & $216 \pm 25$
\end{tabular}

Female, $n=10,19$ eyes

Mean士SD

$\begin{array}{cl}9 \pm 8 & 0.13 \\ 14.7 \pm 1.18 & 0.27 \\ 15.8 \pm 1.13 & 0.46 \\ 19.6 \pm 0.72 & 0.09 \\ 0.23 \pm 0.10 & 0.30 \\ 0.31 \pm 0.12 & 0.66 \\ 53.5 \pm 2.82 & 0.97 \\ 60.7 \pm 2.95 & 0.12 \\ 227 \pm 34.3 & 0.08\end{array}$

CC: Choriocapillaris flow area; CMT: Central macular thickness; DCP: Deep capillary plexus; FAZd: Deep foveal avascular zone area; FAZs: Superficial foveal avascular zone area; SCP: Superficial capillary plexus;VDd:Vascular density in DCP;VDs:Vascular density in SCP. 
Table 2. Correlation between age and coherence tomography angiography parameters

\begin{tabular}{lcc} 
Characteristics & $\mathbf{R}$ & $\mathbf{p}$ \\
\hline CC flow area, $\mathrm{mm}^{2}$ & 0.31 & 0.025 \\
SCP flow area, $\mathrm{mm}^{2}$ & 0.13 & 0.323 \\
DCP flow area, $\mathrm{mm}^{2}$ & 0.14 & 0.317 \\
FAZs, $\mathrm{mm}^{2}$ & -0.06 & 0.67 \\
FAZd, $\mathrm{mm}^{2}$ & -0.14 & 0.32 \\
VDs, \% & 0.08 & 0.58 \\
VDd, \% & 0.21 & 0.13 \\
CMT, $\mu \mathrm{m}$ & 0.01 & 0.92 \\
\hline
\end{tabular}

CC: Choriocapillaris flow area; CMT: Central macular thickness; DCP: Deep capillary plexus; FAZd: Deep foveal avascular zone area; FAZs: Superficial foveal avascular zone area; R: Correlation coefficient; SCP: Superficial capillary plexus;VDd:Vascular density in DCP;VDs:Vascular density in SCP.

related with the FT $(p=0.03)$, and was negatively correlated with the FAZd $(p=0.019)$. These correlations prompted examination of relationships the parameters of FT, FAZ, and VD. Linear regression analysis was performed using the FT as the dependent variable. The analysis revealed a significant association between the FT and the FAZs and FAZd $(p<0.00 \mathrm{I})$ and the VDd $(p=0.007)$. Therefore, it appears that the FT is a critical parameter that affects both the macular VD and the FAZ. The results of the analysis can be seen in Table 4.

\section{Discussion}

Evaluation of structural changes and vasculature alteration in the eye is of great importance in all age groups and populations. However, prior to the development of sophis- ticated noninvasive instruments, detailed eye testing and evaluation was a major hurdle. The retinal structure and vasculature were studied using established invasive procedures, such as indocyanine green angiography and fluorescein angiography (FA). OCTA is noninvasive and can be utilized for various disease conditions, including macular degeneration, open-angle glaucoma, diabetic retinopathy, and blood vessel occlusion.

This study generated a set of normative values for OCTA parameters in a healthy pediatric population. We found that the CC flow area was positively correlated with age and the result was found to be statistically significant. It may be that the CC flow area increases with age; however, to generalize this outcome, further analysis with larger and diverse cohorts is required. Only 2 studies were found that have analyzed OCTA parameters in healthy in children using the RTVue-XR Angiovue device (7,9). Zhang et al. (9) analyzed the FAZd, FAZs, VDs, VDd, and CC. They did not find a correlation between these parameters with age, while Borrelli et al. (7) analyzed the same parameters and found a negative correlation between age and CC. The differences in the findings may be a result of ethnicity, sample size, age distribution, or the measurement method. The measurements evaluated in this study and that of Zhang et al. (9) were automatically provided by the software. Borrelli et al. (7) used ImageJ image analysis software (version 1.50; Schneider, C. A.; Rasband, W. S.; Eliceiri, K. W.; US National Institutes of Health and the Laboratory for Optical and Computational Instrumentation, Bethesda, MD, USA).

The effect of sex and age on the FAZ are not yet clear. Zhang et al. (9) and Niestrata-Ortiz et al. (6) reported that the FAZ was not correlated with age; however, they found that it was significantly related to sex. In contrast, Borelli et

Table 3. Correlation analysis of coherence tomography angiography parameters

\begin{tabular}{|c|c|c|c|c|c|c|}
\hline \multirow[t]{2}{*}{ OCTA parameters } & \multicolumn{2}{|c|}{ CMT } & \multicolumn{2}{|c|}{ FAZs } & \multicolumn{2}{|c|}{ FAZd } \\
\hline & $\mathbf{R}$ & $\mathbf{p}$ & $\mathbf{R}$ & $\mathbf{p}$ & $\mathbf{R}$ & $\mathbf{p}$ \\
\hline SCP flow area, $\mathrm{mm}^{2}$ & 0.32 & 0.018 & -0.50 & $<0.001$ & -0.40 & $<0.001$ \\
\hline DCP flow area, $\mathrm{mm}^{2}$ & 0.39 & 0.04 & -0.53 & $<0.001$ & -0.61 & $<0.001$ \\
\hline $\mathrm{CC}$ flow area, $\mathrm{mm}^{2}$ & -0.02 & 0.9 & -0.13 & 0.336 & -0.19 & 0.154 \\
\hline VDs, \% & -0.07 & 0.62 & -0.03 & 0.82 & -0.09 & 0.479 \\
\hline VDd, \% & 0.29 & 0.03 & -0.256 & 0.064 & -0.32 & 0.019 \\
\hline FAZs, $\mathrm{mm}^{2}$ & -0.65 & $<0.001$ & & & 0.85 & $<0.001$ \\
\hline $\mathrm{FAZd}, \mathrm{mm}^{2}$ & -0.57 & $<0.001$ & 0.85 & $<0.001$ & & \\
\hline
\end{tabular}

CC: Choriocapillaris flow area; CMT: Central macular thickness; DCP: Deep capillary plexus; FAZd: Deep foveal avascular zone area; FAZs: Superficial foveal avascular zone area; OCTA: Optical coherence tomography angiography; SCP: Superficial capillary plexus;VDd:Vascular density in DCP;VDs:Vascular density in SCP. 
Table 4. Univariate linear regression analysis to examine the association between central macular thickness and foveal avascular zone area and vascular density

\begin{tabular}{lccc} 
Parameters & Coefficient & $\mathbf{R}^{2}$ & $\mathbf{p}$ \\
\hline FAZs, mm ${ }^{2}$ & -0.004 & 0.37 & $<0.001$ \\
FAZd, $\mathrm{mm}^{2}$ & -0.003 & 0.347 & $<0.001$ \\
VDs, \% & -0.006 & 0.004 & 0.663 \\
VDd, \% & 0.036 & 0.136 & 0.007 \\
\hline
\end{tabular}

FAZd: Deep foveal avascular zone area; FAZs: Superficial foveal avascular zone area;VDd:Vascular density in DCP;VDs:Vascular density in SCP.

al. (7) found a positive correlation with age but no correlation with sex. Hsu et al. (8) reported no significant correlation between the FAZ and age or sex in adults and children. Our findings were consistent with those of Hsu et al. (8): we observed no effect of age or sex on the FAZ.

We found that the FAZs and FAZd were negatively correlated to the FT $(p<0.001)$. The VDd was positively correlated with the FT $(p=0.03)$ and was negatively correlated with the FAZd $(p=0.019)$. The FT would appear to be a critical factor that affects both the macular VD, and the FAZ. Similarly, Zhang et al. (9) reported that the FT was significantly positively correlated with VD at the DCP. In addition, they also reported a positive correlation between the FT and SCP VD. The lack of significance in our study may be due to the small number of eyes studied. Borrelli et al. (7) also investigated the relationship between the FT and the SCP and DCP density, but did not find a statistically significant association. An inverse relationship between the FAZ and the $\mathrm{FT}$ in adults and children has been reported previously $(5,7,10)$. One hypothesis stated that FAZ maturation is a critical step in the development of the foveal depression, and that hypoxia in the fovea results in apoptosis or a migration of neurons from the FAZ (II). Thus, a larger FAZ may be associated with a thinner FT.

The most important limitation of this study is that the FAZ value was provided automatically by the OCTA software. It is possible that FAZ boundaries drawn manually with image processing software would be more accurate. However, only excellent-quality images and analyses were included in this research to compensate for this potential limitation. Also, a longitudinal follow-up and repeated measures are necessary to better evaluate the effect of age on various parameters. Despite these limitations, the results of this study are still noteworthy.

In conclusion, we submit an initial set of normative values for OCTA parameters in a healthy Turkish pediatric population. Our results suggested no statistically significant effect of sex on OCTA parameters for the healthy subjects evaluated $(p<0.05)$. We found an association between the $C C$ and age, as well as significant associations between the FT and the FAZ and VD. The statistical significance determined strongly supports the relationships presented in this analysis. Nonetheless, analysis of a larger cohort and a longitudinal study with a diverse population are warranted to confirm these findings. Detailed knowledge of the normal quantities of the vascular plexus density, flow, and other parameters measured by OCTA will help to better understand the pathophysiological basis of vascular diseases of the retina.

\section{Disclosures}

Ethics Committee Approval: The study protocol was reviewed and approved by the institutional ethics committee of Kahramanmaras Sutcu Imam University (Protocol no: 235/31.07.2019). This study was conducted in accordance with the Declaration of Helsinki.

Peer-review: Externally peer-reviewed.

Conflict of Interest: None declared.

Authorship Contributions: Involved in design and conduct of the study $(Y K, A C)$; preparation and review of the study $(Y K, A B$, DY.); data collection (AC, $A B$ ); and statistical analysis (YK, DY).

\section{References}

I. Bazvand F, Mirshahi R, Fadakar K, Faghihi H, Sabour S, Ghassemi $F$. The quantitative measurements of vascular density and flow area of optic nerve head using optical coherence tomographyangiography. J Glaucoma 2017;26:735-4I. [CrossRef]

2. lafe NA, Phasukkijwatana N, Chen X, Sarraf D. Retinal capillary density and foveal avascular zone area are agedependent: quantitative analysis using optical coherence tomography angiography. Invest Ophthalmol Vis Sci 2016;57:5780-7. [CrossRef]

3. Veronese C, Maiolo C, Huang D, Jia Y, Armstrong GW, Morara $M$, et al. Optical coherence tomography angiography in pediatric choroidal neovascularization. Am J Ophthalmol Case Rep 2016;2:37-40. [CrossRef]

4. Hautz W, Golebiewska J, Kocyla-Karczmarewicz B. Optical coherence tomography and optical coherence tomography angiography in monitoring Coats' disease. J Ophthalmol 2017;201 7:7849243. [CrossRef]

5. Falavarjani KG, Shenazandi H, Naseri D, Anvari P, Kazemi P, Aghamohammadi F, et al. Foveal avascular zone and vessel density in healthy subjects: an optical coherence tomography angiography study. J Ophthalmic Vis Res 2018;13:260-5. [CrossRef]

6. Niestrata-Ortiz M, Fichna P, Stankiewicz W, Stopa M. Sex-related variations of retinal and choroidal thickness and fove-al avascular zone in healthy and diabetic children assessed by optical coherence tomography imaging. Ophthalmologica 2019;24I:173-8. [CrossRef]

7. Borrelli E, Lonngi M, Balasubramanian S, Tepelus TC, Baghdasaryan E, lafe NA, et al. Macular microvascular networks in healthy pediatric subjects. Retina 2019;39:1216-24. [CrossRef] 
8. Hsu ST, Ngo HT, Stinnett SS, Cheung NL, House RJ, Kelly MP, et al. Assessment of macular microvasculature in healthy eyes of infants and children using OCT angiography. Ophthalmology 2019;126:1703-II. [CrossRef]

9. Zhang Z, Huang $X$, Meng $X$, Chen T, Gu Y, Wu Y, et al. In vivo assessment of macula in eyes of healthy children 8 to 16 years old using optical coherence tomography angiography. Sci Rep 2017;7:8936. [CrossRef]
10. Samara WA, Say EA, Khoo CT, Higgins TP, Magrath G, Ferenczy $\mathrm{S}$, et al. Correlation of foveal avascular zone size with foveal morphology in normal eyes using optical coherence tomography angiography. Retina 2015;35:2 I88-95. [CrossRef]

II. Sandercoe TM, Geller SF, Hendrickson AE, Stone J, Provis JM. VEGF expression by ganglion cells in central retina before formation of the foveal depression in monkey retina: evidence of developmental hypoxia. J Comp Neurol 2003;462:42-54. [CrossRef] 